Lutz Eric Lehmann

Klaus-Peter Hunfeld

Martina Steinbrucker

Volker Brade

Malte Book

Harald Seifert

Tobias Bingold

Andreas Hoeft

Heimo Wissing

Frank Stüber

\section{Improved detection of blood stream pathogens by real-time PCR in severe sepsis}

Received: 24 August 2008

Accepted: 26 June 2009

Published online: 15 September 2009

(C) Springer-Verlag 2009

L. E. Lehmann and K-P. Hunfeld contributed equally to the work.

H. Wissing and F. Stüber conceived equally as senior authors of the study.

L. E. Lehmann (®) - A. Hoeft

Klinik und Poliklinik für Anästhesie und Operative Intensivmedizin,

Universitätsklinik Bonn, Sigmund-Freud-

Str. 25, 53105 Bonn, Germany

e-mail: lutz.lehmann@ukb.uni-bonn.de

Tel.: +49-228-28711557

Fax: +49-228-28716754

K.-P. Hunfeld · M. Steinbrucker · V. Brade Institut für Medizinische Mikrobiologie und Krankenhaushygiene, Universitätsklinikum Frankfurt am Main, Paul-Ehrlich Str. 40, 60596 Frankfurt, Germany

\section{K.-P. Hunfeld}

Zentralinstitut für Laboratoriumsmedizin, Krankenhaus Nordwest, Steinbacher Hohl 2-26, 60488 Frankfurt am Main, Germany

\section{H. Seifert}

Institut für Medizinische Mikrobiologie, Immunologie und Hygiene, Klinikum der Universität zu Köln, Goldenfelsstr. 19-21, 50935 Cologne, Germany
T. Bingold $\cdot$ H. Wissing Zentrum der Anästhesiologie und Wiederbelebung, Universitätsklinikum Frankfurt am Main, Theodor-Stern-Kai 7, 60596 Frankfurt am Main, Germany

\section{E. Lehmann - M. Book · F. Stüber}

Universitätsklinik für Anästhesiologie und Schmerztherapie, Inselspital,

3010 Bern, Switzerland
Abstract Objective: Evaluation of the technical and diagnostic feasi- bility of commercial multiplex real- time polymerase chain reaction (PCR) for detection of blood stream infections in a cohort of intensive care unit (ICU) patients with severe sepsis, performed in addition to con- ventional blood cultures.
Design: Dual-center cohort study. Setting: Surgical ICU of two uni- versity hospitals. Patients and participants: One hundred eight critically ill patients fulfilling the American College of Chest Physi- cians/Society of Critical Care Medicine (ACCP/SCCM) severe sepsis criteria were included. Inter- ventions: None. Measurements and results: $\quad$ PCR results obtained in 453 blood samples from 108 patients were compared with corresponding blood culture results. PCR resulted in a twofold higher positivity rate when compared with conventional blood culture (BC) testing (114 versus 58

positive samples). In 40 out of 58 PCR positive assays the results of the corresponding blood cultures were identical to microorganisms detected by PCR. In 18 samples PCR and BC yielded discrepant results. Compared with conventional blood culture the sensitivity and specificity of PCR was 0.69 and 0.81 , respectively. Further evaluation of PCR results against a constructed gold standard including conventional microbiological test results from other significant patient specimen (such as bronchio-alveolar lavage fluid, urine, swabs) and additionally generated clinical and laboratory information yielded sensitivity of 0.83 and specificity of 0.93 . Conclusions: Our cohort study demonstrates improved pathogen detection using PCR findings in addition to conventional blood culture testing. PCR testing provides increased sensitivity of blood stream infection. Studies addressing utility including therapeutic decision-making, outcome, and cost-benefit following diagnostic application of PCR tests are needed to further assess its value in the clinical setting.

Keywords Detection of blood stream pathogens - Real-time PCR . Blood culture $\cdot$ Severe sepsis 


\section{Introduction}

Sepsis is the second leading cause of death in the noncoronary ICU. Sepsis is associated with mortality rates ranging from $20 \%$ to $50 \%$ [1, 2]. Early diagnosis followed by prompt appropriate treatment [3] improves the prognosis of septic patients $[4,5]$. One important therapeutical aspect is early initiation of calculated antibiotic therapy. Each hour of delay in administration of antibiotics is associated with an average $8 \%$ decrease in survival rate of septic shock [6]. Blood cultures (BC) must be obtained before antibiotic therapy [3] which should be reassessed on the basis of culture results and clinical data [7]. Timely and continuous reassessment is important, since inappropriate antibiotic therapy deteriorates outcome $[8,9]$, whereas adequate therapy is associated with favorable outcome $[10,11]$.

Typically, BC specimens become positive within 24-36 h after sampling and therapy can be optimized based on presumptive bacterial identification. A complete microbial identification and susceptibility profile, however, is usually not available before $24-72 \mathrm{~h}$ later. Despite advances in $\mathrm{BC}$ techniques $[12,13] \mathrm{BC}$ positivity rates remain low and may vary significantly, depending on severity of sepsis and ongoing antibiotic treatment [14-16]. It has been suggested that nucleic-acid-based technology such as PCR is more sensitive and can also shorten the time to result when compared with conventional $\mathrm{BC}$ technique [17]. The development and initial evaluation of such an assay was recently reported $[18,19]$. Our study objective was to evaluate the technical and diagnostic feasibility of a commercial multiplex realtime PCR-based method for diagnosis of blood stream infections in a cohort of ICU patients with severe sepsis. Under the conditions of this investigation PCR was used in addition to conventional $\mathrm{BC}$ diagnostics as performed according to the local standard procedures of the participating centers. Primary endpoint of this study was the positivity of findings for microbial DNA in relation to results from conventional microbiological testing of patients with severe sepsis. Secondary endpoints were differences of Sequential Organ Failure Assessment (SOFA) score, procalcitonin (PCT) and interleukin (IL)-6 plasma levels, length of stay (ICU and hospital), and survival.

\section{Materials and methods}

Patient cohort

This study was performed in patients from two surgical ICUs, including a total of 108 patients, covering a total of 453 paired blood samples, consisting of simultaneously obtained BC and PCR blood samples. The relevant institutional or regional review boards or ethics committees approved the research protocol and participants or legal guardians gave written informed consent. All patients included were clinically suspected of suffering from severe sepsis of bacterial or fungal origin. Severe sepsis was classified according to the ACCP/SCCM consensus conference criteria of 1998 [14]. Inclusion followed after independent decision of the physician in charge to call for a blood culture. Repeated samples per patient were allowed. The Systemic Inflammatory Response Syndrome (SIRS) criteria [temperature, heart rate, respiratory rate, and white blood cell (WBC) count] were obtained for all patients. Further data were compiled regarding antibiotic regimen, site of infection, SOFA score (degree of organ dysfunction), procalcitonin (PCT) and interleukin-6 (IL-6) plasma level, length of stay (ICU and hospital), and hospital survival. Following the prospective and observational study design, physicians in charge did not use PCR test results to guide clinical treatment.

\section{$\mathrm{BC}$ procedures and blood collection for the PCR test}

Twenty microliters whole blood, freshly drawn according to common standards of the German Society of Medical Microbiology and Hygiene (DGHM) [20], were used for a pair of aerobic/anaerobic BC bottles (BACTEC, Becton Dickinson $\mathrm{GmbH}$, Germany). Immediately after drawing blood for culture, $9 \mathrm{ml}$ whole blood was then collected in sterile MONOVETTE ${ }^{\circledR}$ ethylenediamine tetraacetic acid (EDTA) K2E tubes (Sarstedt, Germany) for further analysis by PCR test. All BC were processed using semiautomated BC systems (BACTEC 9240, Becton Dickinson $\mathrm{GmbH}$, Germany) according to the manufacturer's instructions and the published standards of the DGHM [20]. The BC system and the local laboratory software automatically registered time to positive BC.

\section{Sample preparation and PCR procedure}

Preparation of DNA and PCR testing was performed from $1 \mathrm{ml}$ whole blood samples using the SeptiFast ${ }^{\circledR}$ Lys Kit, the SeptiFast ${ }^{\circledR}$ Prep Kit and the LightCycler ${ }^{\circledR}$ SeptiFast Kit as described recently in more detail [18]. In brief, samples were mechanically lyzed and internal controls (IC) were included in each sample and in negative controls (NC). Manual extraction was performed to obtain a final extraction volume of $200 \mu \mathrm{l}$ DNA. Eluate $(50 \mu \mathrm{l})$ was used for subsequent real-time PCR amplification using the LightCycler 2.0 Instrument. Potential contaminations were eliminated using uracil- $N$-glycosylase. DNA amplification targets were conserved and variable parts of the internal transcribed sequence (ITS) regions of bacteria and fungi [18]. Amplified variable parts of any amplification products were then hybridized to genus- or species- 
specific oligonucleotide probes and subjected to softwareassisted temperature melting-peak $\left(T_{\mathrm{m}}\right)$ analysis using the SeptiFast ${ }^{\circledR}$ Software set V2.0 (Roche Diagnostics GmbH, Penzberg, Germany) to reliably identify microorganisms covered by the SeptiFast test panel [18]. A result was regarded as a true negative only if included ICs were measured positive. Moreover, the assay was regarded as valid only if the NC tested negative and the corresponding controls (reagent control and the IC of the NC) were detected within their assigned $T_{\mathrm{m}}$ ranges. According to recent study data, time to report for the method's workflow is less then $6 \mathrm{~h}$ and the analytical sensitivity of the assay ranges between 3 and $100 \mathrm{CFU} / \mathrm{ml}$, depending on the individual microorganism [18].

\section{Comparison of LightCycler ${ }^{\circledR}$ SeptiFast test results} with $\mathrm{BC}$ findings

A blood stream infection was defined as a positive BC result, obtained and analyzed as set forth by the current DGHM procedures [20]. Interpretation of BC findings was performed according to established clinical and microbiological standards (DGHM) [20]. Whether microorganisms identified by PCR represented true infection or contamination was evaluated retrospectively by taking into account the identity of the microorganism detected and by comparing PCR results with corresponding BC findings. For common facultative and obligate bacterial pathogens except potential skin contaminants such as coagulase-negative Staphylococcus (CoNS) and some streptococcal species, a positive BC result was interpreted as evidence for ongoing blood stream infection (BSI). For CoNS and streptococci, positive $\mathrm{BC}$ results were interpreted as evidence for BSI only if BC demonstrated growth of identical organisms in two or more different samples of a given patient within $72 \mathrm{~h}$. If a single positive result was reported for these microorganisms within a 72-h period the results were interpreted as probable contamination. Purpose of this study was primarily to evaluate the technical and diagnostic feasibility of PCR as an add-on to conventional BC diagnostics as routinely performed in the participating centers, no 24-h 7-days-a-week PCR service was provided throughout the investigation. Therefore, direct comparison of "time-to-result" performance between PCR and conventional blood culture methods is not part of the analysis as presented in this study.

\section{Analysis of clinical data in conjunction with PCR} and $\mathrm{BC}$ findings

Sepsis patients were grouped according to BC and PCR results obtained during the patient's individual course. Negative findings throughout the clinical course by both
$\mathrm{BC}$ and PCR led to inclusion in the PCR negative/BC negative group, positive findings by $\mathrm{BC}$ and $\mathrm{PCR}$ led to inclusion in the PCR positive/BC positive group, respectively. Single positive results for PCR without corresponding $\mathrm{BC}$ culture findings throughout the entire course led to inclusion in the PCR positive/BC negative group. Conversely, negative PCR and positive $\mathrm{BC}$ were grouped as PCR negative/BC positive. Maximum PCT level and SOFA score, and length of ICU and hospital stay for each patient's course, as well as patient survival were also recorded for this analysis.

Comparison of LightCycler ${ }^{\circledR}$ SeptiFast test results with a constructed gold standard

In the course of microbiological screening for potential infectious foci in the individuals with severe sepsis additional microbiological samples were taken from various body sites and subjected to conventional culture and additional microbiological testing. For assessment of the diagnostic plausibility of available PCR results, the status of infection in the sepsis cohort was further analyzed in the light of a constructed gold standard generated from such supplementary data from additional microbiological samples. For this in-depth analysis a positive PCR result from the patient's blood was regarded as a true positive only if the detected microorganisms were present also in the corresponding $\mathrm{BC}$ and/or in other supplementary materials of the patient expected to be sterile under healthy conditions (i.e., bronchio-alveolar lavage fluid, intra-abdominal swabs, urine, etc.) obtained \pm 2 days from the onset of the septic episode.

Statistical methods

Comparisons were made by $2 \times 2$ contingency tables. Differences between groups were analyzed by one-way analysis of variance (ANOVA) using the GraphPad Prizm 4.0 statistical software package.

\section{Results}

Study and control population characteristics

A total of 108 patients with severe sepsis from the two study sites were included in the study. All patients were under antibiotic coverage at the beginning and throughout the observational study. The corresponding demographics are depicted in Table 1. The most common underling causes of severe sepsis were peritonitis, pneumonia, and severe sepsis following cardiovascular surgery (Table 1). Most frequent comorbidities were chronic renal failure 
Table 1 Characteristics and causes of sepsis of the study patients

\begin{tabular}{|c|c|c|c|}
\hline & All & Female & Male \\
\hline Patients $(n)$ & 108 & 36 & 72 \\
\hline Age, mean (range), years & $58.37(18-84)$ & $60.10(19-84)$ & $57.42(18-84)$ \\
\hline Survival $(n)$ & $65(60 \%)$ & $25(69 \%)$ & $40(56 \%)$ \\
\hline Nonsurvival $(n)$ & $43(40 \%)$ & $11(31 \%)$ & $32(44 \%)$ \\
\hline Underlying cause of sepsis & Number & & \\
\hline $\begin{array}{l}\text { Abdominal sepsis (peritonitis, necrotizing pancreatitis, } \\
\text { cholangitis or cholecystitis) }\end{array}$ & 35 & & \\
\hline Sepsis following cardiovascular surgery & 28 & & \\
\hline Pneumonia/ARDS & 23 & & \\
\hline Tissue infection following trauma & 15 & & \\
\hline Osteomyelitis & 2 & & \\
\hline Genitourinary infection & 2 & & \\
\hline Mediastinitis & 2 & & \\
\hline Catheter related & 1 & & \\
\hline Total & 108 & & \\
\hline
\end{tabular}

Table 2 Chronic comorbidities of study patients $(n=108)$

\begin{tabular}{lcc}
\hline & ${\text { Total }(\%)^{\mathrm{a}}}^{\mathrm{N}^{\mathrm{a}}}$ & \\
\hline Neoplasms (acute or chronic lymphoma, acute or chronic leukemia, metastatic solid cancer) & 20 & 18.5 \\
Liver failure (biopsy-proven cirrosis, documented variceal hemorrhage or & 18 & 16.7 \\
$\quad$ portal hypertension, hepatic ascites or hepatic encephalopathy) & 55 & 50.9 \\
New York Heart Association class III or IV heart failure & 49 & 21.4 \\
Chronic renal failure (serum creatinine $>1.5$ of upper normal limit) or renal replacement therapy & 19.4
\end{tabular}

${ }^{a}$ Patients $(n=108)$ presented multiple comorbidities. Sums of individual comorbidities $(n=163)$ and relative comorbidity fractions of the whole study population are given

and New York Heart Association class III or IV heart failure (Table 2).

\section{Analysis of PCR compared with BC}

PCR results of 453 blood samples were compared with the results of conventional $\mathrm{BC}$ that had been obtained in parallel at each individual time point of sampling. In septic patients, BC were positive in 58 samples (12.8\%). In the concomitantly drawn EDTA blood samples PCR yielded 114 positive results (25.2\%; Table 3$)$. PCR thus resulted in a twofold higher recovery rate compared with conventional BC (Fig. 1a). Of 58 positive BC findings, 40 corresponded directly to the PCR test results. Based on this, the negative predictive value of PCR versus blood culture as a gold standard was 0.95 , sensitivity was 0.69 , and specificity was 0.81 . However, 18 samples of positive $\mathrm{BC}$ showed different PCR results.

\section{Analysis of discrepancies}

Thirteen out of 18 discrepant cases (72\%) could be resolved either by not being in the panel covered by PCR such as Pantoea agglomerans $(n=4)$ and Proprionibacterium spp. $(n=2)$, or because they were judged as contaminating skin flora ( $n=7$; Table 3$)$. However, five positive BC yielding growth of Serratia marcescens $(n=1)$, Enterococcus faecalis $(n=1)$, Enterococcus faecium $(n=2)$, and Candida glabrata $(n=1)$ did not match the corresponding PCR result (Table 3). Despite evidence of real infection as judged by independent clinical review the negative PCR finding remained unresolved.

Analysis of PCR results versus a constructed gold standard

PCR results were also analyzed against a constructed microbiological gold standard. This constructed gold standard included BC findings and/or additional microbiological test results from other patient materials expected to be sterile under healthy conditions and obtained \pm 2 days from onset of the corresponding septic episode. The rationale for introducing such a constructed gold standard was to further elucidate the plausibility of the unopposed positive PCR. PCR detected 74 pathogens which were not confirmed by the corresponding $\mathrm{BC}$ 
analysis. However 51 of these 74 PCR results matched microbiological test results from additional specimens. These additional results included detection of Enterobacter cloacaelaerogenes $(n=6)$, Escherichia coli $(n=5)$, Klebsiella oxytocalpneumoniae $(n=7)$, Proteus mirabilis $(n=1)$, Pseudomonas aeruginosa $(n=5)$, Serratia marcescens $(n=1)$, Stenotrophomonas maltophilia $(n=1)$, Enterococcus faecalis $(n=6)$, Enterococcus faecium $(n=8)$, Staphylococcus aureus $(n=3)$, Streptococcus spp. $(n=1)$, Aspergillus fumigatus $(n=4)$, and Candida albicans $(n=2)$ by both PCR and conventional culture from primarily sterile body materials other than blood (Table 3). When compared with the constructed gold standard the sensitivity of PCR increased to 0.83 , the specificity increased to 0.93 , and the negative predictive value to 0.95 .

\section{Analysis of the clinical course of the study population}

The 108 sepsis patients were grouped according to matching results of $\mathrm{BC}$ and PCR results during their clinical course. Positive $\mathrm{BC}$ findings with concomitant positive PCR results ( $n=40$ samples) were present in 16 out of 108 patients during the septic episode. PCT plasma level, SOFA score, length of ICU, and hospital stay in these patients were significantly increased, while survival was decreased, when compared with the 66 patients of the cohort with negative findings in $321 \mathrm{BC}$ and parallel PCR samples $(p<0.05$; Table 4$)$.

\section{Discussion}

In this observational study we investigated the implementation and potential add-on utility of a new PCR test compared with conventional BC diagnostics. To date, BC still play a pivotal role in the diagnosis of sepsis and are regarded as the microbiological gold standard for the detection of bloodstream infections in patients with clinical sepsis and fever of unknown origin [17]. The practical value of $\mathrm{BC}$ in the diagnosis of sepsis, however, is clearly impaired due to lengthy detection time. Commonly the positive yield does not exceed 5-30\% in septic patients, mainly depending on the severity of the disease and localization of the infection [21]. Likewise, the sensitivity of $\mathrm{BC}$ is known to be poor for many slow-growing and fastidious organisms [22]. As shown in this study PCRbased assay technology holds promise to circumvent some methodological drawbacks of conventional culture and to enhance detection of bacteria and fungi in patients with suspected sepsis. Compared with conventional BC diagnostics, PCR testing resulted in a twofold higher positivity

Table 3 Microbiological findings by blood culture and PCR

\begin{tabular}{|c|c|c|c|c|}
\hline Gram-negative organisms & BC only & $\mathrm{BC}+\mathrm{PCR}$ & PCR only & PCR + suppl. cultures \\
\hline Enterobacter cloacae or aerogenes & 0 & 7 & $13^{\mathrm{a}}$ & 6 \\
\hline Escherichia coli & 0 & 3 & 16 & 5 \\
\hline Klebsiella oxytoca or pneumoniae & 0 & 1 & $14^{\mathrm{a}}$ & 7 \\
\hline Pantoea agglomerans & 4 & Not in panel & Not in panel & 0 \\
\hline Proprionibacterium spp. & 2 & Not in panel & Not in panel & 0 \\
\hline Proteus mirabilis & 0 & 0 & 1 & 1 \\
\hline Pseudomonas aeruginosa & 0 & 1 & 6 & 5 \\
\hline Serratia marcescens & 1 & 1 & 5 & 1 \\
\hline Stenotrophomonas maltophilia & 0 & 2 & 3 & 1 \\
\hline Gram-positive organisms & BC only & $\mathrm{BC}+\mathrm{PCR}$ & PCR only & $\mathrm{PCR}+\mathrm{MiBi}$ \\
\hline Coagulase-negative Staphylococci & $7^{\mathrm{b}}$ & 7 & $12^{\mathrm{c}}$ & 0 \\
\hline Enterococcus faecalis & 1 & 8 & 15 & 6 \\
\hline Enterococcus faecium & 2 & 2 & 10 & 8 \\
\hline Staphylococcus aureus & 0 & $\overline{5}$ & 8 & 3 \\
\hline Streptococcus spp. & 0 & 0 & 1 & 1 \\
\hline Yeast/fungi & $\mathrm{BC}$ & $\mathrm{BC}+\mathrm{PCR}$ & PCR & $\mathrm{PCR}+\mathrm{MiBi}$ \\
\hline Aspergillus fumigatus & 0 & 0 & 4 & 4 \\
\hline Candida albicans & 0 & 3 & 5 & 2 \\
\hline Candida crusei & 0 & 0 & 1 & 0 \\
\hline Candida glabrata & 1 & 0 & 0 & 0 \\
\hline$\sum$ of all & 18 & 40 & 114 & 51 \\
\hline
\end{tabular}

${ }^{a}$ Indistinguishable for PCR method due to ITS-sequence homologies between E. cloacae and E. aerogenes, and between $K$. oxytoca and $K$. pneumoniae, respectively

b samples of coagulase-negative Staphylococci were regarded as contamination

c Seven further positive PCR results were regarded as contamination and not included 
A

\begin{tabular}{|c|c|c|c|c|}
\cline { 2 - 5 } \multicolumn{2}{c|}{} & \multicolumn{3}{c|}{ Blood culture } \\
\cline { 2 - 5 } \multicolumn{2}{c|}{} & Positive & Negative & $\Sigma$ \\
\hline \multirow{3}{*}{\begin{tabular}{c} 
品 \\
\cline { 2 - 5 }
\end{tabular}} & Positive & 40 & 74 & 114 \\
\cline { 2 - 5 } & Negative & 18 & 321 & 339 \\
\cline { 2 - 5 } & $\Sigma$ & 58 & 395 & 453 \\
\hline
\end{tabular}

\begin{tabular}{|c|c|c|c|c|}
\hline B & & \multicolumn{3}{|c|}{ Sepsis cohort $(n=108)$} \\
\hline & & \multicolumn{3}{|c|}{ Constructed Gold Standard } \\
\hline & & Positive & Negative & $\Sigma$ \\
\hline \multirow{3}{*}{$\frac{0}{0}$} & Positive & 91 & 23 & 114 \\
\hline & Negative & 18 & 321 & 339 \\
\hline & $\Sigma$ & 109 & 334 & 453 \\
\hline
\end{tabular}

Fig. 1 a Analysis of PCR results compared with BC findings in sepsis patients (study group). b Analysis of PCR results compared with a constructed gold standard, comprising positive $\mathrm{BC}$ findings or detected organisms in additional patient specimen (e.g., bronchio-alveolo lavage, urine, swabs)

rate in our cohort of patients with clinical sepsis (Fig. 1a). To further investigate the clinical plausibility of PCR results, we also included additional laboratory data and culture results obtained from additional body sites that were clinically incriminated to be focus of ongoing infection in these patients (constructed gold standard, Fig. 1b). Interestingly, in 51 of 74 conventional samples (69\%) the additional microbiological findings paralleled the PCR results obtained directly from the blood of the corresponding patients (Fig. 1b) and, thus, may be interpreted as an important additional clue for the involvement of the recovered microorganisms in the ongoing septic infection in these individuals. Finally, the remaining 23 PCR-positive but discrepant constructed gold standard-negative samples remain unresolved at this point. Such inconclusive results may be interpreted as being either false-positive PCR assays or false-negative constructed gold standard findings. False-positive PCR results, however, may also originate from the amplification of free DNA released from nonviable or killed bacteria and fungi, thereby mimicking ongoing infection. Future interventional trials utilizing PCR-guided therapy may enlighten the relevance of PCRpositive but $\mathrm{BC}$ (or constructed gold standard) negative findings in the clinical context of the ICU.

Timely and adequate antimicrobial treatment is key for improved outcome in patients with pneumonia, meningitis, and sepsis $[8,23]$. Recently Kumar et al. showed that from the onset of hypotension in patients presenting with septic shock, each hour of delay in adequate antimicrobial treatment on average was associated with $8 \%$ decrease in survival rate [6]. Similarly, improved survival in sepsis patients with early adequate therapy has been demonstrated in several other studies [4, 9-11]. The diagnosis of bacteraemia by $\mathrm{BC}$, however, can be delayed for 12-48 h even in common rapid growers depending on individual growth kinetics and initial inoculum, thereby complicating a more timely diagnosis in patients with sepsis. Moreover, the recovery rate of conventional cultures is clearly impaired after initiation of antimicrobial treatment despite current $\mathrm{BC}$ systems having been modified in an attempt to reduce the effect of antimicrobials in the BC bottle [24]. Although a comparative time-to-result analysis of PCR and BC was not part of this study, the given turnaround time of $6 \mathrm{~h}$ for the PCR assay [18] may turn out to be an important add-on of PCR technology compared with the 12-48 h time interval necessary to generate initial $\mathrm{BC}$ findings $[25,26]$. Another possible advantage of a DNA-based detection system is that the microorganisms causing sepsis do not have to be viable at the time of sampling, thereby also including phagocytized or partly digested bacteria within white blood cells. Thus, PCR may be advantageous particularly in patients receiving antibiotics.

Clearly, little is known about the kinetics and clinical and therapeutic relevance of bacterial DNA present in the blood of patients with sepsis over time in the course of an invasive blood stream infection. The potential influence of circulating bacterial DNA on the severity and outcome of blood stream infections, however, is underlined by the findings of our study demonstrating that PCT plasma levels and SOFA scores were significantly higher, length of ICU and hospital stay were longer, and survival was

Table 4 Clinical data of patients grouped according to PCR and blood culture findings

\begin{tabular}{llllll}
\hline & $\begin{array}{l}\text { Mean PCT } \\
(\mathrm{ng} / \mathrm{ml})\end{array}$ & $\begin{array}{l}\text { Mean SOFA } \\
(\text { Score })\end{array}$ & $\begin{array}{l}\text { LOS-ICU } \\
(\text { days })\end{array}$ & $\begin{array}{l}\text { LOS-hospital } \\
\text { (days) }\end{array}$ & $\begin{array}{l}\text { Survival } \\
(\text { rate })\end{array}$ \\
\hline PCR-/BC- $(n=66)$ & $1.7(0-56.0)$ & $11(2-20)$ & $17(1-89)$ & $23(1-93)$ & 66 \\
PCR+/BC+ $(n=16)$ & $12.1(0.4-139.9)$ & $16(12-20)$ & $36(8-87)$ & $38(8-90)$ & 33 \\
\hline
\end{tabular}

$L O S$-ICU length of stay on ICU, LOS hospital length of stay in hospital 
clearly decreased in a subgroup of patients revealing positive findings in both PCR testing and BC.

Although rapid and specific, an important limitation of the current PCR assay, however, arises from the fact that some pathogens that are not part of the detection panel, such as Pantoea agglomerans and Proprionibacterium spp., were missed by PCR but recovered by BC. In five other cases microorganisms that are part of the PCR detection panel were not detected by PCR but were detected in BC. These cases comprised Serratia marcescens $(n=1)$, Enterococcus faecalis $(n=1)$, Enterococcus faecium $(n=2)$, and Candida glabrata $(n=1)$. Of note, all bacterial isolates in the BC became positive after prolonged culture periods, clearly pointing to a low-level in vivo bacteraemia at the time point of sampling. The results of quantitative BC studies have shown that most episodes of clinically significant bacteraemia in adults are characterized by low numbers of circulating bacteria. Werner et al. found that $54 \%$ of BC from adult patients with staphylococcal and streptococcal endocarditis contained between 1 and $30 \mathrm{CFU} / \mathrm{ml}$ of blood [27]. Moreover, Kreger et al. described that $73 \%$ of 77 patients with Gram-negative bacteremia had BC that contained $<10 \mathrm{CFU} / \mathrm{ml}$ of blood [28]. An analytical assay sensitivity of 3-30 CFU/ml [18] and a predefined detection panel of 25 different BSI pathogens, thus, limit to some extent the diagnostic capabilities of the current assay in patients with sepsis when compared with a theoretical sensitivity of one CFU per culture bottle in conventional blood culture after inoculating $\sim 10 \mathrm{ml}$ whole blood. Increasing the starting volume of the PCR test may help to increase the stochastic probability of picking up a pathogen in cases of low-level bacteraemia. In addition, the current test could be improved by adding an additional assay component for the detection of consensus DNA of bacteria or fungi, thereby providing identification of pathogens which are not primarily part of the PCR panel. The feasibility of assays diagnosing bacteria and fungi by consensus DNA detection has been described previously [29].

Summing up, the novel PCR-based assay system, at present, cannot fully substitute conventional $\mathrm{BC}$ also because a more general susceptibility testing of the recovered organisms is not yet possible. Nevertheless, the clinical value of classical BC techniques was also clearly impaired, in part due to the low recovery rate in our sepsis cohort. Moreover, BC show a prolonged time-to-result interval, as it usually takes at least $12-48 \mathrm{~h}$ to provide the clinician with Gram stain results and species identification.

As shown in the present study, novel real-time PCRbased molecular biological assays for rapid detection of important pathogens causing BSI can overcome some limitations of the conventional culture-based microbiological techniques, especially when it comes to more rapid detection and species identification of blood stream pathogens. As such, molecular test systems may be seen as a valuable add-on, providing important additional information to the treating physicians very early in the course of disease, thereby potentially tailoring the antibiotic coverage in septic patients. Whether the assay will indeed significantly contribute to more rapid initiation of better-tailored antimicrobial therapy and improved patient management in conjunction with other laboratory markers, however, awaits further evaluation of the test in interventional clinical studies.

\section{Conclusions}

Our study demonstrates good concordance of PCR results with BC. Moreover, we confirmed the increased sensitivity of PCR-based detection of bloodstream infections, as shown in other studies. These results are indicative of potential clinical utility of PCR-based pathogen identification in patients with severe sepsis. Studies addressing changes in clinical decision-making, outcome, and costbenefit by the utilization of PCR results, however, are needed.

Acknowledgments Rebecca Rödel and Sabine Mering provided expert technical assistance.

Conflict of interest statement The authors have no competing interests. The authors, however, received research funding, reagents, and equipment from Roche Diagnostics $\mathrm{GmbH}$ for this project.

\section{References}

1. Martin GS, Mannino DM, Eaton S, Moss M (2003) The epidemiology of sepsis in the United States from 1979 through 2000. N Engl J Med 348:1546-1554
2. Angus DC, Linde-Zwirble WT, Lidicker J, Clermont G, Carcillo J, Pinsky MR (2001) Epidemiology of severe sepsis in the United States: analysis of incidence, outcome, and associated costs of care. Crit Care Med 29:1303-1310
3. Dellinger RP, Carlet JM, Masur H, Gerlach H, Calandra T, Cohen J, Gea-Banacloche J, Keh D, Marshall JC, Parker MM, Ramsay G, Zimmerman JL, Vincent JL, Levy MM (2004) Surviving Sepsis Campaign guidelines for management of severe sepsis and septic shock. Crit Care Med 32:858-873 
4. Gao F, Melody T, Daniels DF, Giles S, Fox S (2005) The impact of compliance with 6-hour and 24-hour sepsis bundles on hospital mortality in patients with severe sepsis: a prospective observational study. Crit Care 9:R764-R770

5. Rivers E, Nguyen B, Havstad S, Ressler J, Muzzin A, Knoblich B, Peterson E, Tomlanovich M (2001) Early goaldirected therapy in the treatment of severe sepsis and septic shock. N Engl J Med 345:1368-1377

6. Kumar A, Roberts D, Wood KE, Light B, Parrillo JE, Sharma S, Suppes R, Feinstein D, Zanotti S, Taiberg L, Gurka D, Kumar A, Cheang M (2006) Duration of hypotension before initiation of effective antimicrobial therapy is the critical determinant of survival in human septic shock. Crit Care Med 34:1589-1596

7. Bochud PY, Bonten M, Marchetti O, Calandra T (2004) Antimicrobial therapy for patients with severe sepsis and septic shock: an evidence-based review. Crit Care Med 32:S495-S512

8. Harbarth S, Nobre V, Pittet D (2007) Does antibiotic selection impact patient outcome? Clin Infect Dis 44:87-93

9. Ibrahim EH, Sherman G, Ward S, Fraser VJ, Kollef MH (2000) The influence of inadequate antimicrobial treatment of bloodstream infections on patient outcomes in the ICU setting. Chest 118:146-155

10. Garnacho-Montero J, GarciaGarmendia JL, Barrero-Almodovar A, Jimenez-Jimenez FJ, Perez-Paredes C, Ortiz-Leyba C (2003) Impact of adequate empirical antibiotic therapy on the outcome of patients admitted to the intensive care unit with sepsis. Crit Care Med 31:2742-2751

11. Leibovici L, Shraga I, Drucker M, Konigsberger H, Samra Z, Pitlik SD (1998) The benefit of appropriate empirical antibiotic treatment in patients with bloodstream infection. $\mathbf{J}$ Intern Med 244:379-386

12. Cockerill FR 3rd, Wilson JW, Vetter EA, Goodman KM, Torgerson CA, Harmsen WS, Schleck CD, Ilstrup DM, Washington JA 2nd, Wilson WR (2004) Optimal testing parameters for blood cultures. Clin Infect Dis 38:1724-1730
13. Magadia RR, Weinstein MP (2001) Laboratory diagnosis of bacteremia and fungemia. Infect Dis Clin North Am 15:1009-1024

14. American College of Chest Physicians/ Society of Critical Care Medicine Consensus Conference Committee (1992) American College of Chest Physicians/Society of Critical Care Medicine Consensus Conference: definitions for sepsis and organ failure and guidelines for the use of innovative therapies in sepsis. Crit Care Med 20: 864-874

15. Rangel-Frausto MS, Pittet D, Costigan M, Hwang T, Davis CS, Wenzel RP (1995) The natural history of the systemic inflammatory response syndrome (SIRS). A prospective study. JAMA 273:117-123

16. Sands KE, Bates DW, Lanken PN, Graman PS, Hibberd PL, Kahn KL, Parsonnet J, Panzer R, Orav EJ, Snydman DR, Black E, Schwartz JS, Moore R, Johnson BL Jr, Platt R (1997) Epidemiology of sepsis syndrome in 8 academic medical centers. JAMA 278:234-240

17. Peters RP, van Agtmael MA, Danner SA, Savelkoul PH, VandenbrouckeGrauls CM (2004) New developments in the diagnosis of bloodstream infections. Lancet Infect Dis 4:751-760

18. Lehmann LE, Hunfeld KP, Emrich T, Haberhausen G, Wissing H, Hoeft A, Stuber F (2008) A multiplex real-time PCR assay for rapid detection and differentiation of 25 bacterial and fungal pathogens from whole blood samples. Med Microbiol Immunol 197:313-324

19. Louie RF, Tang Z, Albertson TE, Cohen S, Tran NK, Kost GJ (2008) Multiplex polymerase chain reaction detection enhancement of bacteremia and fungemia. Crit Care Med 36:1487-1492

20. Seifert H, (2001) MiQ: Qualitätsstandards in der mikrobiologisch-infektiologischen Diagnostik Heft 3: SepsisBlutkulturdiagnostik. Elsevier, Urban \& Fischer
21. Calandra T, Cohen J (2005) The international sepsis forum consensus conference on definitions of infection in the intensive care unit. Crit Care Med 33:1538-1548

22. Socan M, Marinic-Fiser N, Kese D (1999) Comparison of serologic tests with urinary antigen detection for diagnosis of legionnaires' disease in patients with community-acquired pneumonia. Clin Microbiol Infect 5:201-204

23. Kollef MH, Sherman G, Ward S, Fraser VJ (1999) Inadequate antimicrobial treatment of infections: a risk factor for hospital mortality among critically ill patients. Chest 115:462-474

24. Dellit TH, Owens RC, McGowan JE Jr, Gerding DN, Weinstein RA, Burke JP, Huskins WC, Paterson DL, Fishman NO, Carpenter CF, Brennan PJ, Billeter M, Hooton TM (2007) Infectious Diseases Society of America and the Society for Healthcare Epidemiology of America guidelines for developing an institutional program to enhance antimicrobial stewardship. Clin Infect Dis 44:159-177

25. Harbarth S, Garbino J, Pugin J, Romand JA, Lew D, Pittet D (2003) Inappropriate initial antimicrobial therapy and its effect on survival in a clinical trial of immunomodulating therapy for severe sepsis. Am J Med 115:529-535

26. Struelens MJ, de Mendonca R (2001) The emerging power of molecular diagnostics: towards improved management of life-threatening infection. Intensive Care Med 27:1696-1698

27. Werner AS, Cobbs CG, Kaye D, Hook EW (1967) Studies on the bacteremia of bacterial endocarditis. JAMA 202:199-203

28. Kreger BE, Craven DE, McCabe WR (1980) Gram-negative bacteremia. IV. Re-evaluation of clinical features and treatment in 612 patients. Am J Med 68:344-355

29. Cursons RT, Jeyerajah E, Sleigh JW (1999) The use of polymerase chain reaction to detect septicemia in critically ill patients. Crit Care Med 27:937-940 